\title{
Does propofol sedation increase the cecal intubation rate in colonoscopy?
}

\author{
Ali Tardu, ${ }^{1}$ Zeliha Türkyılmaz, ${ }^{1}$ Makbule Elif Yılmaz, ${ }^{2}$ Gürhan Çelik ${ }^{1}$ \\ 'Department of General Surgery, Edirne State Hospital, Edirne, Turkey \\ 2Department Of Anesthesiology, Edirne State Hospital, Edirne, Turkey
}

\begin{abstract}
Introduction: One of the most important quality indicators in a colonoscopy procedure is intubation of the cecum. The aim of this study was to investigate the effect of propofol sedation on cecal intubation.

Materials and Methods: A total of 186 patients who underwent colonoscopy with propofol-based sedation were examined retrospectively. Seven cases were excluded from the study for various reasons. Patient demographic data, colonoscopy findings, and sedation protocols were recorded.

Results: Of the 179 patients included, 100 were males. The mean age was $54.8 \pm 16.2$ years, and the mean body mass index was $26.6 \pm 3$.6. The average propofol dose was $141.5 \pm 49.1 \mathrm{mg}$. The cecum was intubated in 176 cases $(98.3 \%)$. None of the patients were observed to develop a major complication as a result of the colonoscopy.

Conclusion: In the current study, both the cecal intubation rate, which is higher than what is suggested in the related body of literature, and the fact that no complications were observed in any of the patients could suggest that propofol-based sedation is both safe and effective.

Keywords: Deep sedation; incomplete colonoscopy; reaching cecum.
\end{abstract}

\section{Introduction}

Colonoscopy is the gold standard method in the screening of colorectal cancer, in the evaluation of lower gastrointestinal system diseases, and also in the detection and removal of certain polyps detected during the procedure. However, colonoscopy is an invasive procedure. It causes pain and discomfort in patients. Cecal intubation is one of the main goals of colonoscopy and at the same time, it is one of the quality indicators of colonoscopy. ${ }^{[1-3]}$ The patient's pain during the procedure may prevent the endoscope from reaching the cecum. To prevent this situation, patients may be given conscious or deep sedation. In this retrospective study, the contribution of propofol sedation to cecal intubation is tried to be revealed.

\section{Materials and Methods}

The data of patients who underwent colonoscopy with propofol sedation between November 2016 and May 2017 by a single endoscopist at Edirne State Hospital General Surgery Endoscopy Unit were retrospectively examined. Patients who had previously undergone colorectal surgery, patients with complete obstruction due to colorectal 
tumor, patients with stenosis following distal pelvic surgery, and patients who were immediately ended due to poor colon cleansing were excluded. The patients' gender, age, referral basis, American Society of Anesthesiologists (ASA) score, Body Mass Index (BMI), previous abdominal surgery, sedation protocol, and colonoscopy findings were recorded.

\section{Procedure}

All the patients were treated with polyethylene glycol $\left(\right.$ Golytely ${ }^{\circledR}$ ) for bowel preparation. Anesthesia specialist or technician provided deep sedation without endotracheal intubation with midazolam and propofol. Depending on the depth of sedation, an additional dose of propofol was administered if necessary. After colonoscopy, the patients were monitored until they were fully awake, and they were discharged on the same day.

\section{Results}

186 patients, who underwent colonoscopy between the aforementioned dates, were examined. Seven patients were excluded from the study for various reasons. For instance, two of them had undergone previous surgery due to right colon cancer and rectum cancer. One patient's bowel cleansing was inadequate, and therefore the procedure was not continued. The other two patients were found to have rectal cancer, and in another one right colon cancer was detected. In these patients, the procedure was terminated as the mass could not be passed. The last one had an operative history due to retrorectal mass. Fur- thermore, rectal stenosis was also detected in this patient, and stenosis could not be passed.

Of the remaining patients, 100 were males and 79 were females. The patient's age mean was $54.8 \pm 16.2$ and the BMI mean was $26.6 \pm 3.6 \mathrm{~kg} / \mathrm{m}^{2}$. Their demographic data is given in Table 1. While forty-two patients were performed colonoscopy for screening, 112 patients were performed it for gastrointestinal symptoms. 16 of the patients were performed colonoscopy for control after polypectomy. To the rest, colonoscopy was performed due to foreign body, rectovaginal fistula, and the like (Table 2). All of the patients were provided with deep sedation with midazolam and propofol. The average dose of propofol was $1.94 \pm 0.82 \mathrm{mg} /$ $\mathrm{kg}$. None of the patients needed endotracheal intubation or mask ventilation. In one of them, the procedure was ended as he developed bradycardia during colonoscopy. No major complication such as perforation of the colon or hemorrhage occurred in any of the patients.

176 out of 179 patients were achieved cecal intubation (98.3\%), but 3 patients could not be intubated cecum. In one of these patients, it was not possible to go beyond the mid-transverse colon due to long sigmoid colon and excessive loop formation. For another patient had total abdominal hysterectomy and bilateral salpingo-oophorectomy (TAH + BSO), it was not possible to pass through the splenic flexure in this patient. In the last patient, who could not also be cecal intubated, colonoscopy was not continued because of bradycardia occurred during the procedure.

Table 1. Demographic data of patients

\begin{tabular}{lccc} 
& & \multicolumn{3}{c}{ Cecal intubation rate } \\
\cline { 3 - 5 } & & $\mathbf{n}$ & $\%$ \\
\hline Number of patients (male/female) & $100 / 79$ & & \\
Age, median (range), year & $57(17-87)$ & & \\
BMI $\left(\mathrm{kg} / \mathrm{m}^{2}\right)$ & & & \\
$\quad<25$ & 58 & 56 & 96.5 \\
$\geq 25-<30$ & 87 & 87 & 100 \\
$\quad \geq 30$ & 35 & 34 & 97.1 \\
American Society of Anesthesiologists & & & \\
$\quad$ I & 20 & 20 & 100 \\
$\quad$ II & 61 & 61 & 100 \\
III & 98 & 95 & 96.9 \\
Propofol dose, median (range), (mg/kg) & $1.85(0.53-3.45)$ & & \\
\end{tabular}




\begin{tabular}{lcc}
\hline Table 2. Indication for colonoscopy & & \\
\hline & $\mathbf{n}$ & $\%$ \\
\hline Screening & 42 & 23.5 \\
Post polypectomy & 16 & 8.9 \\
Constipation or diarrhea & 59 & 32.9 \\
Rectal bleeding & 18 & 10.1 \\
Abdominal pain & 26 & 14.5 \\
Other & 18 & 10.1 \\
\end{tabular}

One or more polyps were seen in $52(29.5 \%)$ of the patients who underwent colonoscopy. 31 (17.6\%) of them were adenoma. Colorectal malignancy was detected in nine patients. Two patients had inflammatory bowel disease. Colonic diverticula, foreign body, rectovaginal fistula, etc. were detected in 16 of the patients. No pathology was detected in 97 patients who underwent complete colonoscopy.

\section{Discussion}

In the current study, the cecal intubation rate (CIR) is $98.3 \%$, which is thought to be quite high. Besides, polyp detection rate and adenoma detection rate are $29.5 \%$ and $17.6 \%$ in turn, and are consistent with the related body of literature. The quality of the colonoscopy procedure is evaluated through the pre-procedural quality measures (bowel preparation), procedural measures (CIR, adenoma detection rate, withdrawal time), post procedural measures (surveillance interval), and other quality measures (patient satisfaction and willingness to repeat the procedure). ${ }^{[3,4]}$ Since the study is retrospective, there is no data in hand for such parameters as withdrawal time, patient satisfaction, and willingness to repeat the procedure. Only one in 186 patients had poor bowel cleansing.

International guidelines recommend $90 \%$ CIR in the routine colonoscopy procedure. ${ }^{[4]}$ However, in the literature, it has been reported that the CIR changes between 55\% and $98.8 \% \cdot{ }^{[5]}$ Nonetheless, in colonoscopies performed by experienced endoscopists, cecal intubation failures occur only for $5-10 \%$. ${ }^{[6]}$

The most common causes of incomplete colonoscopy are pain, stenosis, poor bowel preparation, low BMI, advanced age, gender (female), hysterectomy story, diverticulitis, inflammatory bowel disease, loop formation of the intestines, and complication during the procedure. . $^{[3,5,7]}$ In our series, one of the three incomplete processes had hysterectomy story, and the other had loop formation. To increase CIR, various techniques such as water infusion and carbon dioxide insufflation, and also various device modifications like pediatric, robotic, double balloon, long or very thin colonoscopy usage have been reported. ${ }^{[3,8,9]}$

For most of the patients, colonoscopy is a painful and disturbing procedure. Discomfort felt during the procedure is one of the most important reasons for reducing CIR and patient satisfaction. Therefore, in many countries, it is performed under either conscious or deep sedation. ${ }^{[10]}$ In their very comprehensive study including more than 50,000 cases, Bannert et al. ${ }^{[7]}$ have shown that colonoscopy performed under sedation increased CIR in both men and women. Radaelli et al. ${ }^{[1]}$ reported that, CIR in colonoscopies with sedation and without sedation are in turn $84.2 \%$ and $76.1 \%$. This rate is quite low when compared to ours.

The most common sedation strategy during colonoscopy is the use of analgesic-assisted benzodiazepines. ${ }^{[10]}$ In recent years, propofol-based deep sedation has begun to be used as propofol which has been shown to have a better pharmacokinetic profile than benzodiazepines and opioids. ${ }^{[12]}$ Propofol sedation is believed to increase complications during colonoscopy. However, a meta-analysis showed that propofol was safely used even in high-risk patients. ${ }^{[13]}$ In our series, bradycardia was observed to develop in a patient during the procedure. However, we could not detect whether this is due to propofol.

As a result, deep sedation maintained through propofol during colonoscopy is a safe and an effective procedure. As patient's pain and discomfort are minimalized through deep sedation, it increases both patient's satisfaction and endoscopist's comfort. Moreover, propofol sedation can increase CIR. However, the current study is retrospective and included limited number of cases. Therefore, wider and more randomly controlled studies are needed.

\section{Disclosures}

Ethichs Committee Approval: Retrospective study.

Peer-review: Externally peer-reviewed.

Conflict of Interest: None declared.

\section{References}

1. Lieberman DA, Weiss DG, Bond JH, Ahnen DJ, Garewal H, Chejfec G. Use of colonoscopy to screen asymptomatic adults for colorectal cancer. Veterans Affairs Cooperative Study Group 380. N Engl J Med 2000;343:162-8. [CrossRef] 
2. Morini S, Zullo A, Hassan C, Lorenzetti R, Campo SM. Endoscopic management of failed colonoscopy in clinical practice: to change endoscopist, instrument, or both? Int J Colorectal Dis 2011;26:103-8. [CrossRef]

3. Hancock KS, Mascarenhas R, Lieberman D. What Can We Do to Optimize Colonoscopy and How Effective Can We Be? Curr Gastroenterol Rep 2016;18:27. [CrossRef]

4. Rex DK, Schoenfeld PS, Cohen J, Pike IM, Adler DG, Fennerty $\mathrm{MB}$, et al. Quality indicators for colonoscopy. Gastrointest Endosc 2015;81:31-53. [CrossRef]

5. Akere A, Akande KO. Cecal intubation rate during colonoscopy at a tertiary hospital in South-West Nigeria: How frequent and what affects completion rate? Niger $\mathrm{J}$ Clin Pract 2017;20:303-6. [CrossRef]

6. Tumino E, Parisi G, Bertoni M, Bertini M, Metrangolo S, lerardi $E$, et al. Use of robotic colonoscopy in patients with previous incomplete colonoscopy. Eur Rev Med Pharmacol Sci 2017;21:819-26.

7. Bannert C, Reinhart K, Dunkler D, Trauner M, Renner F, Knoflach $P$, et al. Sedation in screening colonoscopy: impact on quality indicators and complications. Am J Gastroenterol 2012;107:1837-48. [CrossRef]

8. Sofi AA, Nawras A, Khan MA, Howden CW, Lee WM. Me- ta-analysis of the performance of ultrathin vs. standard colonoscopes. Endoscopy 2017;49:351-8.

9. Sunada K, Shinozaki S, Yano T, Hayashi Y, Sakamoto H, Lefor AK, et al. Double-Balloon Colonoscopy Has a Higher Cecal Intubation Rate Than Conventional Colonoscopy Using a CoIon Simulator. Dig Dis Sci 2017;62:979-83. [CrossRef]

10. Ferreira AO, Torres J, Barjas E, Nunes J, Glória L, Ferreira R, et al. Non-anesthesiologist administration of propofol sedation for colonoscopy is safe in low risk patients: results of a noninferiority randomized controlled trial. Endoscopy 2016;48:747-53. [CrossRef]

11. Radaelli F, Meucci G, Sgroi G, Minoli G; Italian Association of Hospital Gastroenterologists (AIGO). Technical performance of colonoscopy: the key role of sedation/analgesia and other quality indicators. Am J Gastroenterol 2008;103:1122-30.

12. Rex DK, Deenadayalu VP, Eid E, Imperiale TF, Walker JA, Sandhu K, et al. Endoscopist-directed administration of propofol: a worldwide safety experience. Gastroenterology 2009;137:1229-37. [CrossRef]

13. Wang D, Chen C, Chen J, Xu Y, Wang L, Zhu Z, et al. The use of propofol as a sedative agent in gastrointestinal endoscopy: a meta-analysis. PLoS One 2013;8:e53311. [CrossRef] 